\title{
Optogenetic tools light up phase separation
}

\author{
Researchers have developed two optogenetic tools for probing and controlling phase separation in living cells.
}

A s discussed in our January Methods to Watch section, biologists are discovering that not only membrane-bound organelles but also dynamic, membraneless condensates have critical roles in the spatiotemporal regulation of cellular processes. These intracellular condensates are thought to be formed through liquid-liquid phase separation (LLPS) arising from weak, multivalent interactions that often involve intrinsically disordered proteins or regions (IDPs or IDRs, respectively). It is, however, still largely unknown how these IDPs and their interacting partners precisely control the LLPS process, and how these phase-separated condensates are organized to retain their structural and biological functions.

The challenge arises from the lack of tools that can capture the process of LLPS in the cellular environment. "There really are very limited tools to study LLPS," notes Clifford Brangwynne from Princeton University, who has a long-standing interest in intracellular phase separation and its role in cell function and dysfunction.

In 2009 when Clifford Brangwynne was a postdoc at the Max Planck Institute of Molecular Cell Biology and Genetics, he and his colleagues from Tony Hyman's lab were among the first to reveal the liquid-droplet property of $\mathrm{P}$ granules inside Caenorhabditis elegans. Since then, Brangwynne has been developing tools in combination with soft matter physics to study the biophysical properties of LLPS.

"Primarily, people have been purifying proteins and looking at them in vitro, often under non-physiological conditions, and then trying to infer what it means inside of a cell. The lack of tools is precisely why my group has invested so heavily in developing these new technologies," Brangwynne comments. He and his team now have developed a set of tools that use light to induce biomolecule condensation in living cells.

In two recently published papers, Brangwynne's team describe the independent developments of Corelet, for mapping phase diagrams of local and global phase separation, and CasDrop, which uses the CRISPR-Cas9 system for genometargeting phase separation.

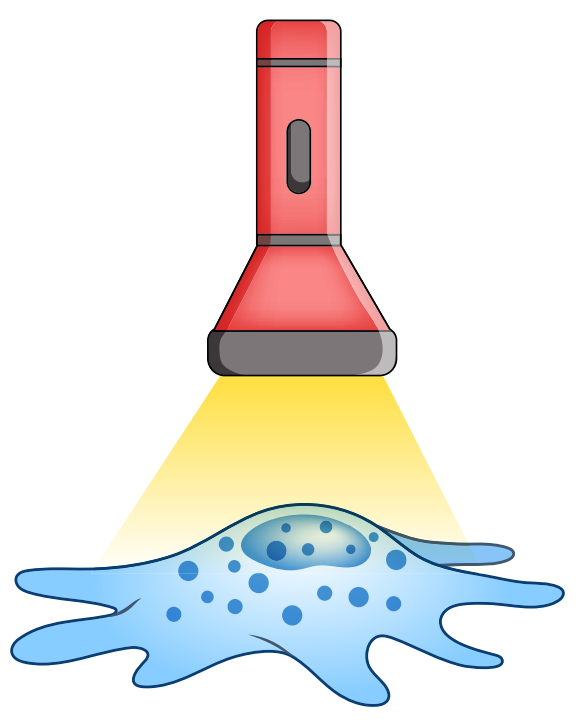

Optogenetic tools shed light on intracellular and nuclear condensates. Credit: Marina Corral Spence/Springer Nature

Corelet includes two modules: the GFPtagged Core functionalized with improved light-inducible dimer (iLID) domains, and the associated binding partners (e.g., SspB) that also contain an mCherry tag and IDR domain. To mimic the native oligomerization process, the Corelet system uses blue light to drive the binding event between iLID and SspB, which in turn generates IDR-containing liquid droplets. This visible, reversible droplet formation provides an opportunity to map intracellular phase diagrams and investigate the changes in phase diagrams caused by protein sequence mutations.

The Corelet system reveals a diffusive capture mechanism in which local light activation can induce multivalent Core to capture and concentrate the associated IDR proteins, and thus result in local phase separation even under globally dilute IDR concentrations. This local phase separation indicates that native droplet condensation may also occur through a local driving force such as protein phosphorylation controlled by spatially patterned kinases or phosphatases.

In related work, Brangwynne, along with his postdoc Yongdae Shin and doctoral student Yi-Che Chang, developed CasDrop, which aims to illuminate the mechanism of liquid condensation at specific genomic loci in the cell nucleus where these condensates manifest as assemblies of the nucleolus, transcriptional clusters, or other nuclear bodies.

The CasDrop system contains two components: (1) dCas9-ST-GFP-iLID, which harnesses CRISPR-dCas9 to target the specific genes and optogenetic dimerization protein iLID like Corelet to generate photoactivatable binding scaffolds for recruiting IDR proteins; and (2) IDR-mCh$\mathrm{SspB}$, the binding partner that induces the formation of IDR oligomers and leads to a localized LLPS at the target genomic loci.

They noticed that in control cells not expressing targeting guide RNA, a variety of IDR-containing liquid condensates preferentially form and grow in low-density chromatin regions and exclude chromatin, which might be explained by the lower energy cost of deforming softer genomic regions.

In the study of targeting condensation, they found that CasDrop can bring distal genomic loci together to form liquid condensates through surface-tension-driven coalescence, while mechanically excluding non-targeted neighboring genomes. On the basis of these findings, they proposed a chromatin filter model for genome restructuring in which nuclear condensates play both filter-out and pull-in roles.

"The next steps are going to require moving beyond the qualitative, correlative descriptions. ... And we'll be able to examine how this phase behavior underlies the huge variety of biological functions that are now associated with intracellular condensed phases, from synaptic vesicle organization to cell division," Brangwynne remarks.

Lei Tang

Published online: 30 January 2019 https://doi.org/10.1038/s41592-019-0310-5

Research papers

Bracha, D. et al. Mapping local and global liquid phase behavior in living cells using photooligomerizable seeds. Cell 175, 1467-1480 (2018). Shin, Y. et al. Liquid nuclear condensates mechanically sense and restructure the genome. Cell 175, 1481-1491 (2018). 